Ophthalmologe 2006 $\cdot 103: 533-536$ DOI 10.1007/s00347-005-1265-8

Online publiziert: 17. September 2005

๑) Springer Medizin Verlag 2005

M. G. Todorova · A. M. Palmowski-Wolfe - D. Leifert · C. Prünte · P. Meyer

Augenklinik, Universität, Basel

\title{
Unilaterale nächtliche Blendempfindung und Photopsien
}

\section{Vorgeschichte}

Eine 61-jährige Patientin stellte sich in unserer Klinik mit seit 2 Wochen bestehenden Photopsien, nächtlicher Blendempfindlichkeit und einem temporalen Gesichtsfeldausfall am rechten Auge vor. Vor 2 Jahren hatte sie sich wegen eines metastasierten amelanotischen Melanoms der Vulva einer Operation unterziehen müssen.

\section{Ophthalmologischer Befund}

Der Fernvisus betrug beidseits 1,0. Der Nahvisus war am rechten Auge auf 0,5 und am linken auf o,6 reduziert. Der Augendruck lag beidseits applanatorisch bei $18 \mathrm{mmHg}$.

Die vorderen Augenabschnitte waren regelrecht. Der Fundusbefund war beidseits bis auf eine leichte Engstellung der retinalen Arterien unauffällig. Am rechten Auge fand sich ein mittelperipherer, flacher Naevus (1 PD Durchmesser). Funktionsprüfungen ergaben eine konzentrische Einengung des Gesichtsfelds rechts (• Abb. 1) sowie eine erhöhte Verwechslungshäufigkeit in der BlauGelb-Achse (Panel D15), die rechts ausgeprägter war.

\section{Elektrophysiologie}

Im Ganzfeld-ERG nach ISCEV-Standard zeigte sich beidseits unter skotopischen Bedingungen eine deutliche Herabsetzung der b-Wellen-Amplitude mit negativem ERG rechts. Gleichzeitig waren die Amplituden der a-Welle am rechten Auge bei normalen Latenzen vermindert
(• Abb. 2). Das photopische ERG lag im Normbereich, wobei die Amplituden im Seitenvergleich rechts kleiner als links waren. Die oszillatorischen Potenziale waren herabgesetzt (• Abb. 3).

Zusätzlich wurde ein multifokales ERG (mfERG; Retiscan) der ON- und OFFReizantwort durchgefürt (ON-Stimulus 106 ms Dauer, OFF-Stimulus 106 ms Dauer) und mit einer Kontrollgruppe $(n=4)$ verglichen.

Die Reizantworten wurden in 3 Gruppen gemittelt und analysiert (Gruppe 1: zentrale $7,5^{\circ}$, Gruppe 2: 7,5-21, $9^{\circ}$, Gruppe 3: $21,9-32,1^{\circ}$ ) und mit einer Kontrollgruppe $(n=4)$ verglichen. Hier zeigte sich, dass sowohl die ON-, als auch die OFF-Amplituden beidseits, insbesondere zentral, herabgesetzt waren (• Abb. 4, Tab. 1).

Um den Einfluss von Amplitudenschwankungen zwischen den Ableitungen zu reduzieren, wurde das Verhältnis der OFF/ON-Antworten gebildet. Dabei resultierte am rechten Auge ein pathologisches OFF/ON-Verhältnis in allen Exzentrizitäten, am linken Auge dagegen nur zentral (• Tab. 1). Die Latenzen der ON-Reizantwort waren am rechten Auge in allen Exzentrizitäten verzögert, am linken Auge dagegen grenzwertig normal. Die OFF-Latenzen beider Augen waren im Normbereich.

Präsentiert auf der 102. Tagung der Deutschen Ophthalmologischen Gesellschaft 2004, Berlin 22.-26.09.2004 


\section{Bild und Fall}

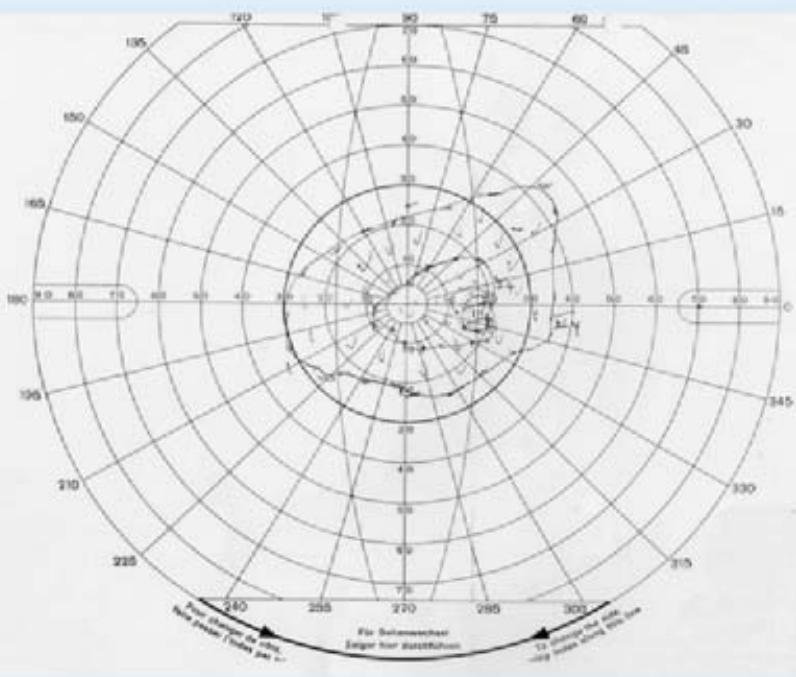

rechtes Auge

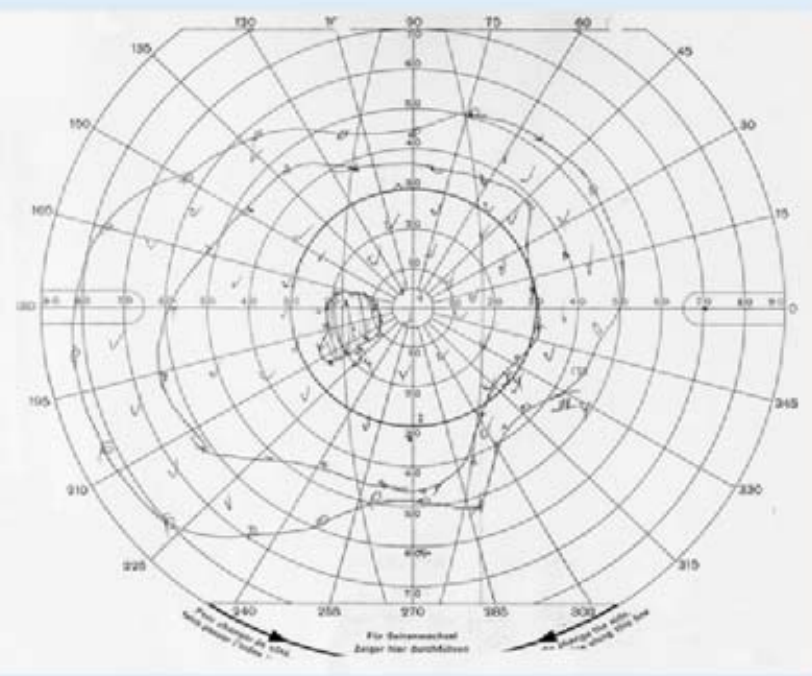

linkes Auge

Abb. $1 \Delta$ Kinetische Goldmann-Perimetrie der Patientin mit konzentrischer Gesichtsfeldeinengung am rechten Auge bei weitestgehend unauffälligem Befund am linken Auge

Intensität

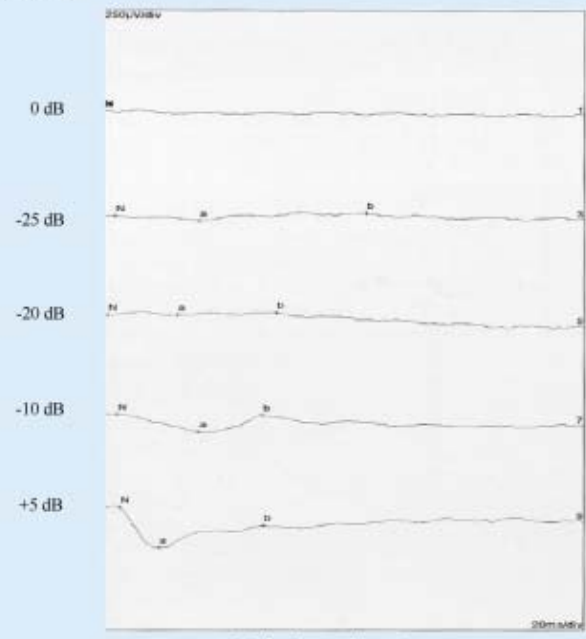

Patientin rechtes Auge
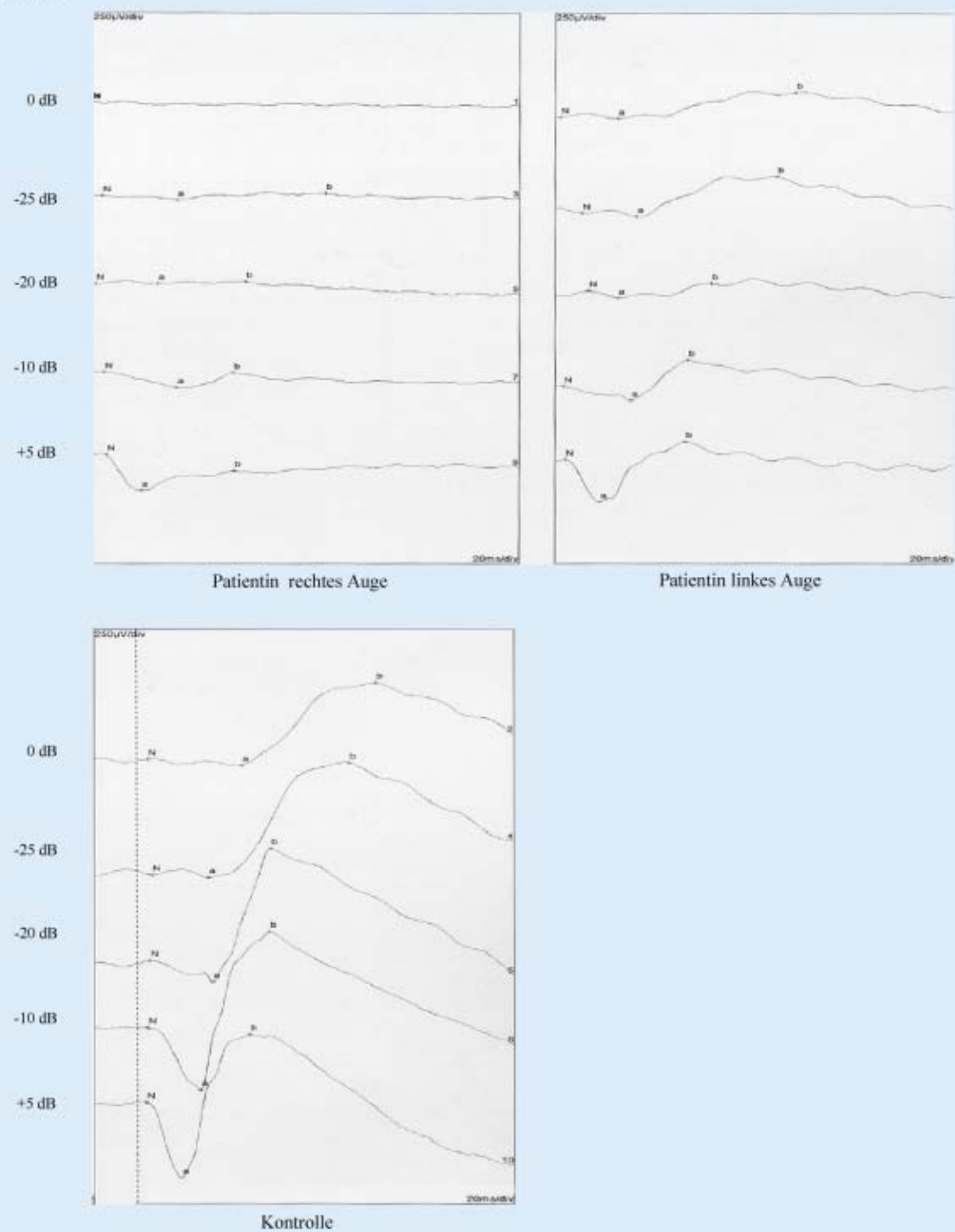

Abb. $2 \Delta$ Skotopisches Ganzfeld-ERG (ISCEV-Standard) der Patientin (oberer Abbildungsteil) und einer repräsentativen Kontrollperson (unterer Abbildungsteil): Bds. im Vergleich Amplitudenminderung der b-Welle mit sog.,"negativem“ ERG des rechten Auges zu erkennen 


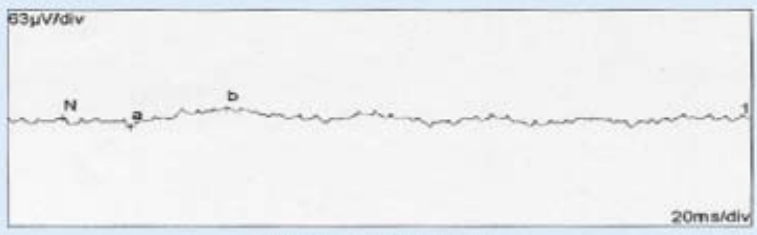

Patientin rechtes Auge

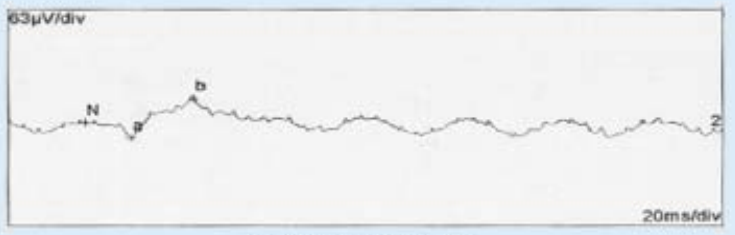

Abb. 3 - Oszillatorische Potenziale (Ausschnitt aus dem Ganzfeld-ERG, ISCEV-

Standard) der Patientin (oberer und mittlerer Abbildungsteil) und einer repräsentativen Kontrollperson (unterer Abbildungsteil): Oszillatorische Potenziale im Vergleich erloschen
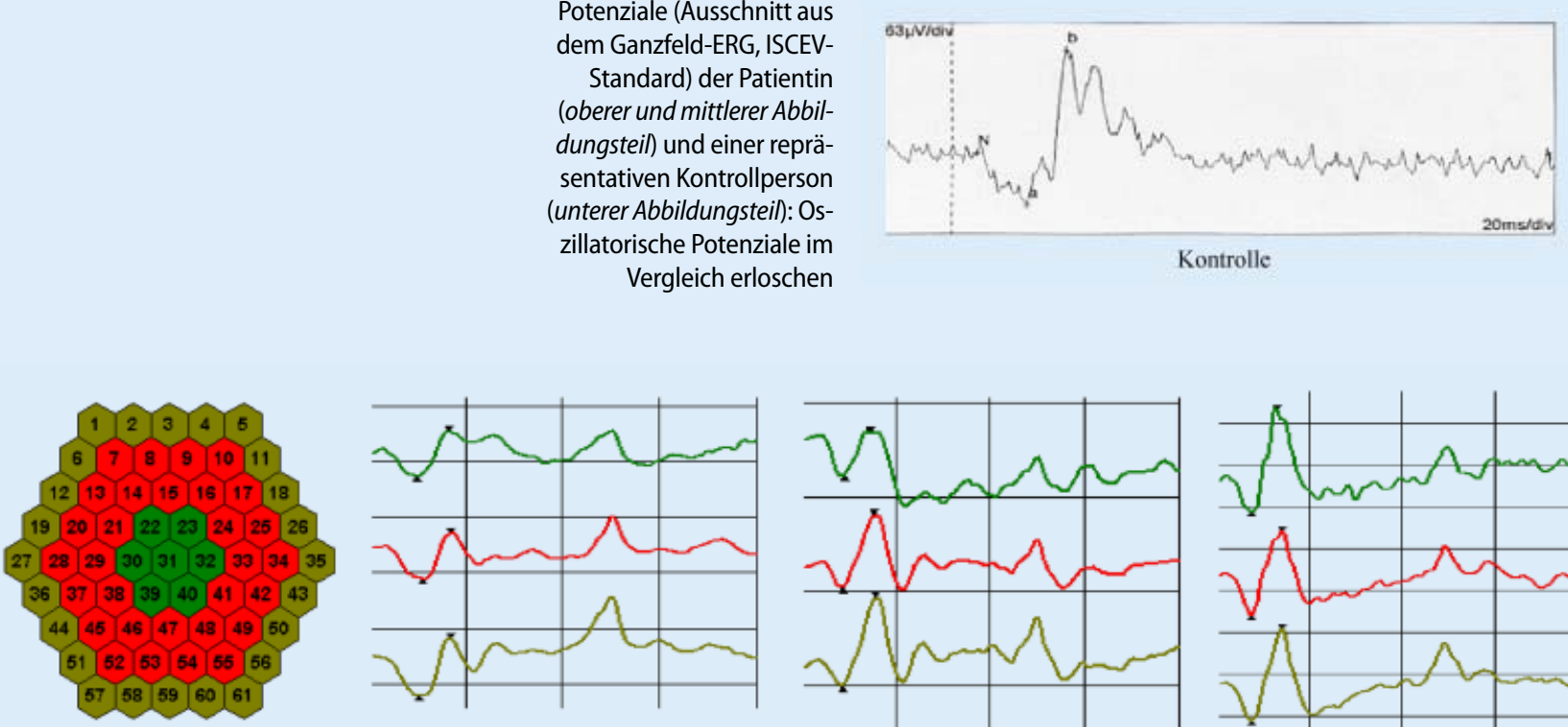

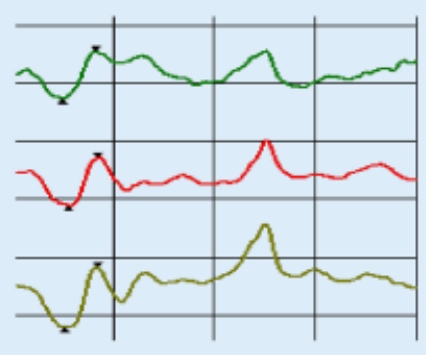

Patientin RA

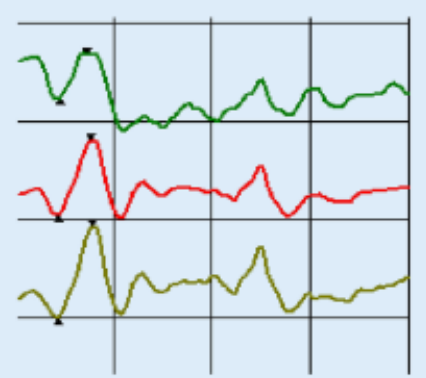

Patientin LA

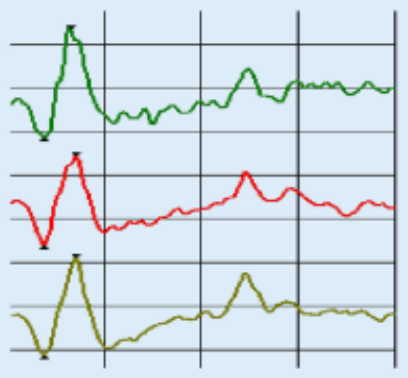

Kontrolle

Abb. 4 Multifokales ON-OFF-ERG der Patientin und einer repräsentativen Kontrollperson mit graphischer Darstellung der Reizantwortgruppierung

\begin{tabular}{|c|c|c|c|c|c|c|}
\hline $\begin{array}{l}\text { Gruppie- } \\
\text { rung der } \\
\text { Reizanwor- } \\
\text { ten }\end{array}$ & $\begin{array}{l}\text { ON-Amp- } \\
\text { lituden } \\
\left.\text { [nV/deg }{ }^{2}\right] \\
\text { MAR }\end{array}$ & $\begin{array}{l}\text { ON-Amp- } \\
\text { lituden } \\
\left.\text { [nV/deg }{ }^{2}\right] \\
\text { Kontrolle }\end{array}$ & $\begin{array}{l}\text { OFF-Amp- } \\
\text { lituden } \\
{\left[\mathrm{nV} / \mathrm{deg}^{2}\right]} \\
\text { MAR }\end{array}$ & $\begin{array}{l}\text { OFF-Amp- } \\
\text { lituden } \\
{\left[\mathrm{nV} / \mathrm{deg}^{2}\right]} \\
\text { Kontrolle }\end{array}$ & $\begin{array}{l}\text { Verhält- } \\
\text { nis der } \\
\text { OFF/ON- } \\
\text { Antworten } \\
\text { MAR }\end{array}$ & $\begin{array}{l}\text { Verhält- } \\
\text { nis der } \\
\text { OFF/ON- } \\
\text { Antworten } \\
\text { Kontrolle }\end{array}$ \\
\hline \multirow[t]{2}{*}{$0-7,5^{\circ}$} & RA 22,5 & \multirow[t]{2}{*}{$46,2-69,0$} & RA 13,8 & \multirow[t]{2}{*}{$19,9-28,7$} & RA 0,61 & \multirow[t]{2}{*}{$0,36-0,62$} \\
\hline & LA 8,6 & & LA 7,2 & & LA 0,83 & \\
\hline \multirow[t]{2}{*}{$7,5-21,9^{\circ}$} & RA 11,9 & \multirow[t]{2}{*}{$24,0-29,0$} & RA 10,2 & \multirow[t]{2}{*}{$8,4-14,6$} & RA 0,85 & \multirow[t]{2}{*}{$0,30-0,54$} \\
\hline & LA 8,5 & & LA 4,0 & & LA 0,45 & \\
\hline \multirow[t]{2}{*}{$21,9-31,1^{\circ}$} & RA 9,7 & \multirow[t]{2}{*}{$20,0-25,0$} & RA 8,1 & \multirow[t]{2}{*}{$9,0-12,8$} & RA 0,83 & \multirow[t]{2}{*}{$0,40-0,59$} \\
\hline & LA 8,6 & & LA 4,2 & & LA 0,48 & \\
\hline \multicolumn{7}{|c|}{ RA: rechtes Auge, LA: linkes Auge. } \\
\hline
\end{tabular}




\section{\ Diagnose: Melanomassoziierte Retinopathie (MAR).}

\section{Diskussion}

Die MAR ist eine paraneoplastische Retinopathie bei Patienten mit malignem Melanom der Haut. Sie ist charakterisiert durch retinale Antikörper gegen depolarisierende Bipolarzellen und geht dementsprechend mit spezifischen funktionellen Störungen der ON-Bipolarzellen einher [1]. Die Patienten klagen typischerweise über vermehrte nächtliche Blendempfindlichkeit und Photopsien.

Untersuchungen können in der Regel eine konzentrische Einengung des Gesichtsfelds, Verwechslungen in der BlauGelb-Achse sowie ein negatives skotopisches ERG als Ausdruck der gestörten Funktion depolarisierender ON-Bipolarzellen aufdecken.

Mit dem mfERG erhalten wir topographische Zusatzinformationen. Beim herkömmlichen mfERG sind reduzierte Amplituden mit verlängerter Latenzzeit durch Störung der ON-Bipolarzellen zu erwarten [2, 3]. Die ON- und OFF-Reizantworten können mittels längerer Stimuli (106 ms, s.o.) besser voneinander differenziert werden. Durch Stimulation mit einem LED-Monitor kann ein Lichtreiz mit einer konstanten Leuchtdichte während der ON-Phase eines Stimulus generiert werden.

Unter Verwendung dieses Ansatzes zeigte sich bei unserer Patientin an beiden Augen eine pathologische ON-Antwort, wobei deren Amplituden im Seitenvergleich links kleiner als rechts waren (• Tab. 1). Da keine umfangreichen Studien über die interokuläre Variabilität des ON-OFF-mfERG vorliegen, haben wir das Verhältnis der OFF/ON-Antworten kalkuliert, um den Einfluss einer interokulären Variabilität zu reduzieren. Am rechten Auge fand sich ein pathologisches $\mathrm{OFF} / \mathrm{ON}$-Verhältnis in allen Exzentrizitäten, während dies am linken Auge nur zentral reduziert war. Dieser elektrophysiologische Befund ist gut vereinbar mit den Beschwerden unserer Patientin: Dies waren beidseits zentrale Funktionseinbußen mit reduziertem Visus und pathologischem Farbensehen, zusätzlich rechts eine ausgedehntere Beeinträchtigung mit nächtlicher Blendungsempfindlichkeit und Photopsien. Ausgelöst durch die elektrophysiologische Diagnostik wurden in der Folge bei unserer Patientin weitere Metastasen des malignen Melanoms gefunden.

Kenntnisse der retinalen Funktion einschließlich des ON-OFF-ERG bei MARPatienten liefern wichtige frühe Hinweise auf den individuellen Verlauf der schwerwiegenden Erkrankung.

Die resultierenden topographischen Informationen der ON-OFF-Antworten können weitere Einblicke in den $\mathrm{Pa}$ thomechanismus der MAR geben. Mehr Informationen bezüglich intertest- und interindividueller Variabilität müssen erhoben werden, bevor Ergebnisse über die absoluten Amplituden in Betracht gezogen werden sollten. Wird das Verhältnis der OFF/ON-Antworten gebildet, verlieren Amplitudenschwankungen zwischen den Ableitungen an Bedeutung. Daher ist dies ein guter Parameter für die Evaluation von Pathologien der ON- und OFF-Bipolarzellen.

Unseres Wissens ist dies der erste Bericht über multifokale, mit Hilfe eines LED-Monitors generierte ON-OFF-Antworten bei MAR.

\section{Korrespondierender Autor \\ M. G. Todorova \\ Augenklinik, Universität \\ Mittlere Straße 91, 4012 Basel \\ TodorovaM@uhbs.ch}

Interessenkonflikt. Der korrespondierende Autor versichert, dass keine Verbindungen mit einer Firma, deren Produkt in dem Artikel genannt ist, oder einer Firma, die ein Konkurrenzprodukt vertreibt, bestehen Unterstützt durch ein Stipendium der Fa. Pfizer (TM, PA).

\section{Literatur}

1. Alexander KRFG, Peachey NS, Marchese AL, Tso MO (1992),On' response defect in paraneoplastic night blindness with cutaneous malignant melanoma. Invest Ophthalmol Vis Sci 33(3):477-483

2. Haus $A H$, Palmowski AM, Pföhler C, Reinhold U et al. (2002) Melanoma-associated retinopathy: screening for melanoma-associated retinopathy in patients with cutaneus malignant melanoma. Neuro-Ophthalmology 27:121-137
3. Hood DC, Frishman LJ, Saszik S, Viswanathan S (2002) Retinal origins of the primate multifocal ERG: implications for the human response. Invest Ophthalmol Vis Sci 43(5):1673-1685 